\title{
High-risk prostate cancer: value of multi-modality 3T MRI-guided biopsies after previous negative biopsies
}

\author{
Jurgen J. Fütterer, ${ }^{1}$ Sadhna Verma, ${ }^{2}$ Thomas Hambrock, ${ }^{1}$ Derya Yakar, ${ }^{1}$ \\ Jelle O. Barentsz ${ }^{1}$ \\ ${ }^{1}$ Department of Radiology (667), Radboud University Nijmegen Medical Centre, Geert Grooteplein 10, 6500 HB Nijmegen, \\ The Netherlands \\ ${ }^{2}$ Department of Radiology, University Of Cincinnati Medical Center, Cincinnati, OH, USA
}

\begin{abstract}
Transrectal ultrasound-guided biopsy is the gold standard for prostate cancer detection. The latter detection rates of random systematic TRUS-guided biopsy do not exceed $44 \%$. As a consequence other biopsy methods have been explored. One of these methods is MR-guided biopsy (MRGB), which revealed detection rates of 38-59\% after previous negative TRUSGB sessions. For this reason MRGB will probably become more and more applied in daily practice.
\end{abstract}

Key words: Prostate cancer-Detection-MR imaging-MR-guided biopsy

The incidence and mortality of prostate cancer vary substantially worldwide, but it is the most common noncutaneous malignancy in the western world. Prostate cancer is the second most common cause of cancer deaths among men in the United States and western Europe [1]. Although there has been a decline in prostate cancer death rates since 1990, attributed to the advent of prostate specific antigen (PSA) leading to earlier diagnosis and treatment, the management of prostate cancer is complicated by our inability to accurately stage disease and predict rate of progression [2]. There are multiple options for treatment which include the following: active surveillance, androgen deprivation therapy, radical prostatectomy (open, laparoscopic, or robotic), various forms of radiation therapy (conformal or intensity modulated external beam and permanent interstitial or

Correspondence to: Jurgen J. Fütterer; email: J.Futterer@rad.umcn.nl high dose rate brachytherapy), or combined approaches $[3,4]$.

Currently in the United States, men undergo screening for prostate cancer beginning at the age of 50 (younger for high risk patients such as African-American or with family history of prostate cancer) using serum PSA combined with digital rectal exam (DRE). Those who have PSA $\geq 4.0 \mathrm{ng} / \mathrm{ml}$ or abnormal DRE are referred for trans-rectal ultrasound (TRUS)-guided prostate biopsy to obtain tissue diagnosis. While prostate biopsy is needed to confirm the diagnosis of prostate cancer, TRUS prostate biopsy is specific (i.e., false positive results are rare), but not sensitive (i.e., 39-52\% false negative rate). This is in distinction to serum PSA which is sensitive $(85 \%$ of patients with prostate cancer have an elevated PSA), but not specific (70-80\% of patients with elevated serum PSA who subsequently undergo TRUSguided prostate biopsy do not have demonstrable prostate cancer) [5, 6]. Clinical staging (e.g., DRE) fails to accurately stage prostate cancer leading to alternative methods of evaluation based upon nomograms (the Partin Tables being the most widely recognized) derived from risk assessment analysis based upon historical analysis of clinically available variables such as serum PSA (and PSA velocity, serum-free PSA), Gleason histologic grading, and surrogate imaging studies such as CT scan and bone scan which assess metastatic but not local disease [7].

Despite its low sensitivity, TRUS-guided prostate biopsy is the universally accepted method of confirming prostate cancer. The term "TRUS-guided" is misleading since TRUS is unable to discriminate between prostate cancer and other benign diseases of the prostate which can cause elevation of serum PSA, the most notable being benign prostate hyperplasia and prostatitis. TRUS 


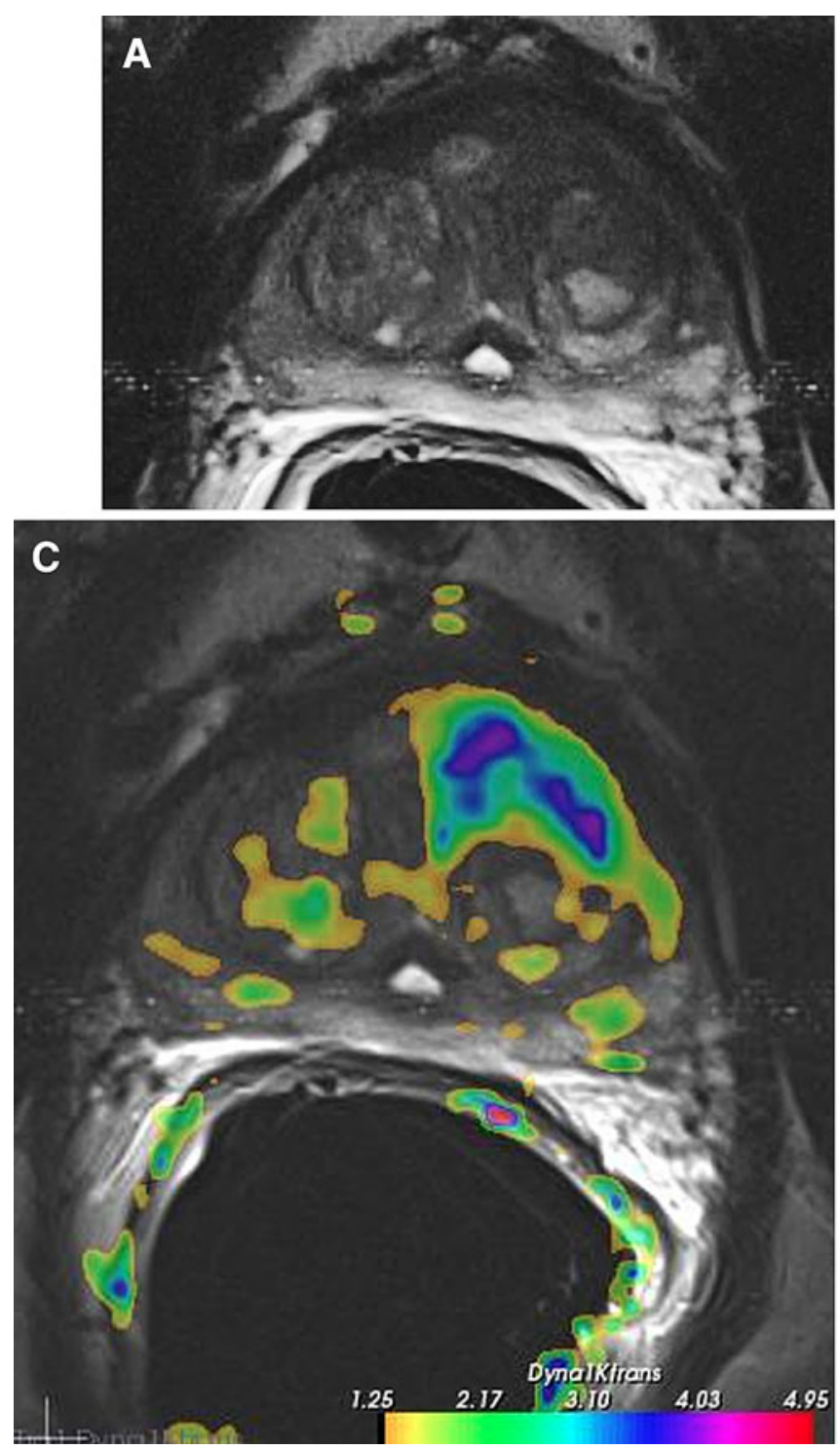

Fig. 1. Multi-parametric MR images, comprising T2-weighted (A), diffusion weighted-derived apparent diffusion coefficient map image (B), and dynamic contrast-enhanced image (Ktrans) (C), in a 63 year old male, with a PSA of $12 \mathrm{ng} / \mathrm{ml}$, and a history of two negative transrectal ultrasound guided prostate biopsy sessions. In the left transitional zone a cancer suspicious region is present (low-signal intensity lesion on T2,

guidance is capable of assuring that the prostate (rather than adjacent organs) is biopsied in a systematic fashion taking two cores from each "sextant" region of the prostate: two biopsies at mid, base, and apex region on both right and left sides (12 needle cores total with variable yield) $[8,9]$. Therefore, men who have a normal TRUS prostate biopsy despite persistent elevated PSA may harbor occult prostate cancer.

Magnetic resonance (MR) imaging has a higher sensitivity for detecting prostate carcinomas than DRE and TRUS in patients having an elevated PSA value and a
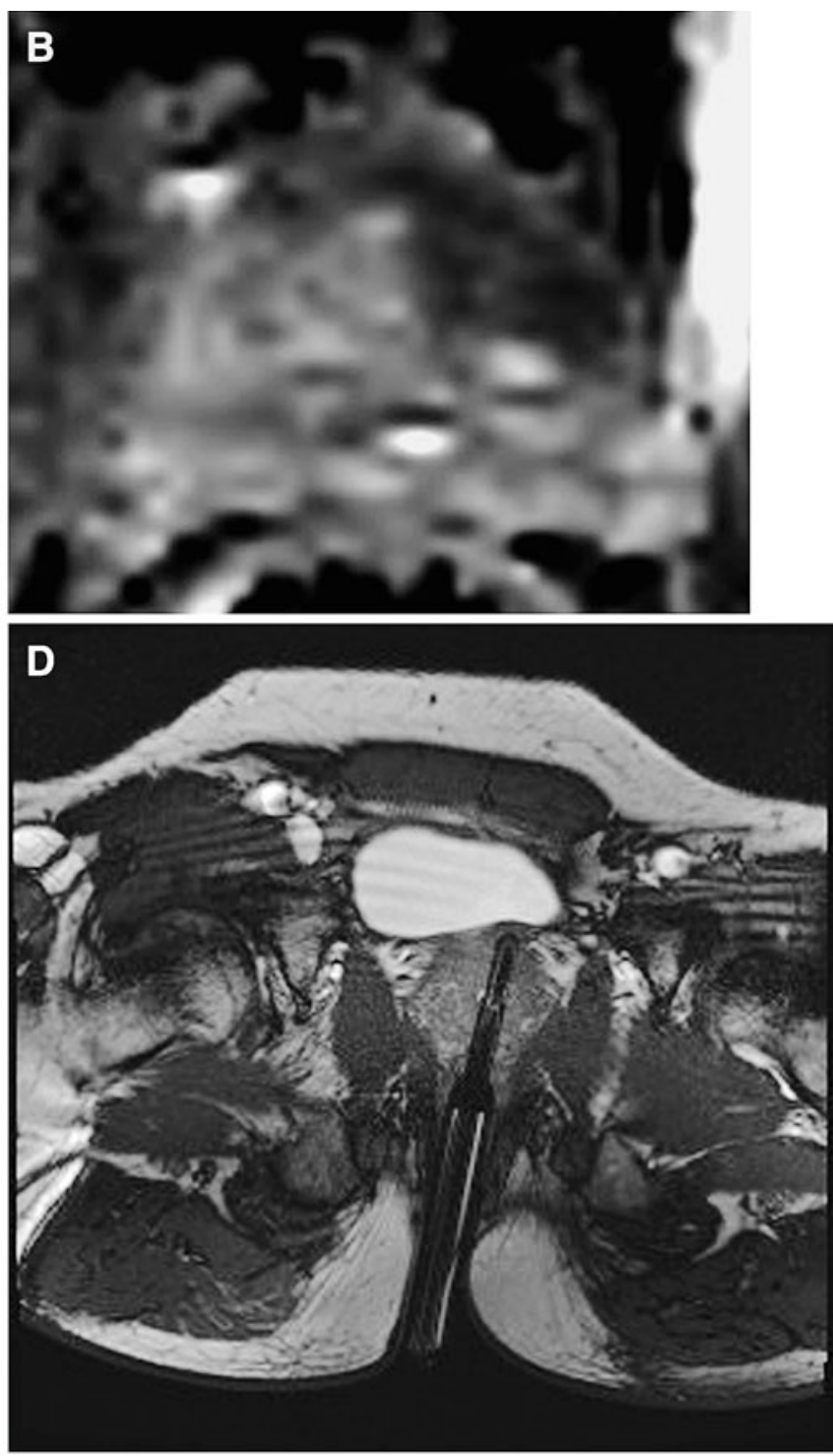

asymmetric Ktrans and restricted diffusion on the ADC map). D During a second session, a MR-guided biopsy was performed of the cancer suspicious area. The needle guider was pointed toward the cancer suspicious region in axial and subsequently biopsied. Verification image with the needle guider left in situ was obtained. Histopathology revealed a Gleason $4+3$ prostate cancer.

negative TRUS biopsy. However, its specificity is limited since other abnormalities such as prostatitis, scars, or prostatic intraepithelial neoplasia (PIN), have a similar MR imaging appearance. However, the role of MRI has evolved over the past decade with development of newer techniques to localize stage and obtain functional information about the tumor.

Currently, prostate MR imaging is the most widely used cross-sectional imaging technique for prostate cancer. While ultrasound provides real-time data, it is also highly operator-dependent and experience is needed 
to perform it. MR imaging allows for a more standardized examination of the prostate and with the addition of functional imaging techniques such as diffusion-weighted imaging (DWI), proton MR spectroscopic imaging (MRSI), and dynamic contrast-enhanced MR imaging (DCE-MRI) a unique insight can be obtained in the cancer characteristics. [10].

Repeat negative biopsies represent a clinical problem faced by urologists. Early detection of significant tumors is crucial to establish effective, potentially curative treatment. Newly developed MR-compatible biopsy devices enable the performance of targeted biopsies in areas that appear suspicious on the MR imaging. Recent studies have demonstrated that MR imaging can be used to target and guide biopsies of suspicious areas seen on a comprehensive diagnostic MR exam [11-16].

Using diagnostic MR images during an MR-guided biopsy procedure improves quality of the biopsy as a high percentage of prostate cancers can be depicted using a targeted biopsy technique, eliminating unnecessary "saturation" prostate biopsies for patients with elevated PSA levels and repeated negative TRUS biopsy sessions. This review will give an overview on the pre-biopsy planning and how to perform MR-guided biopsy (MRGB) of the prostate.

\section{MR imaging}

Prior to the MR-guided biopsy procedure, a diagnostic MR examination is acquired to detect cancer suspicious regions (CSRs). This is typically done in patients with elevated and/ or rising PSA and at least one negative TRUS-guided biopsy session. Either a 1.5 or $3 \mathrm{~T}$ MR scanner is used combined with a multi-phased array coil. The endorectal coil could be used in combination with the phased array coil; however, in this patient group, the aim is to detect the tumor and not to accurately stage prostate cancer.

The imaging protocol should include at least one functional MR imaging techniques. Anatomical T2-weighted turbo spin echo sequences are acquired ( $3 \mathrm{~mm}$ slice thickness; in plane resolution $\leq 0.5 \mathrm{~mm}$ ) in at least two planes (preferably axial and coronal), covering the entire prostate and seminal vesicles. Subsequently, axial DWI is acquired with a single-shot echo-planar imaging sequence with diffusion modules and fat suppression pulses. Water diffusion in at least three directions is acquired using $b$-values of 50,500 , and 800 $1000 \mathrm{~s} / \mathrm{mm}^{2}$. Apparent diffusion coefficient (ADC)-map is calculated based on these $b$-values. In order to perform DCE-MRI, a transverse 2- or 3D T1-weighted gradient echo sequence with the same positioning angle and center as the axial T2-weighted sequence, is acquired ( $>3 \mathrm{~min}$ length and temporal resolution of $\leq 15 \mathrm{~s}$ ) during an intravenous bolus injection of a paramagnetic gadolinium chelate, administered with a power injector at $2-5 \mathrm{ml} / \mathrm{s}$ and followed by a $20-\mathrm{ml}$ saline flush.
The MR images can be evaluated using commercially available analytical software solutions. Prostate cancer typically appears as an area of low-signal intensity within the bright peripheral zone on anatomical T2-weigthed MR imaging. In the central gland, a homogeneous low signal intensity and lenticular shape are significantly associated with the presence of transition zone cancer. Post-biopsy hemorrhage and prostatitis cause areas of low signal intensity on T2-weighted sequences, thereby making prostate cancer detection more difficult. Most practical is to score for every CSR for each different MR parameter the likelihood of cancer being present. A scale of $1-5$ can be used, where a score of 1 indicates no cancer present and a score of 5 indicates that cancer is highly likely to be present. Criteria per imaging sequence for scoring cancer presence in short are; a focal area of low signal intensity on T2-w, on the DCE-MR images a focally enhancing region on the volume transfer constant map and/or washout map, and on the DWI a focally low-signal intensity region on the apparent diffusion coefficient map in combination with a high-signal-intensity region on the image obtained with a high $b$ value (e.g., > 1000).

\section{Patient preparation}

It is advisable to prepare patients preventively with oral or intravenous quinolones. Patients with indications for endocarditis prophylaxis and patients on anti-coagulation medication should be recognized, and if necessary further adjustments should be made depending on hospital protocols. No further preparation is necessary, such as special diets or enemas. Patients should be instructed not to move during the intervention. MRGB can be performed in both open and closed bore systems. At the same time the hindrance of a closed bore system is the limited space one has to position the patient, perform specific maneuvres and handle instruments required for certain interventions. Such problems may be overcome till a certain level with newer MR systems with larger and shorter bores.

As for biopsy routes, the transrectal approach is probably the most preferable one. Even though transgluteal and transperineal biopsy methods have been described and seem feasible, the transrectal approach is the only method where anaesthetics can be omitted [17, 18]. The rectum is the shortest way to reach the prostate and therefore technically the most straightforward approach. Furthermore, it will suffer the least from e.g., needle deflection.

\section{MR-guided biopsy}

Systematic TRUS-guided biopsy is the gold standard for detecting prostate cancer. Initially sextant biopsy schemes were used but these miss a large number of tumors (20-30\%). Increasing the number of cores increased the detection rate on first biopsy from $32,38,42$ to $43 \%$ 
for the 6-, 12-, 18-, and 21-core schemes, respectively. The combination of diagnostic MR imaging and MRGB may increase the prostate cancer detection rate (Fig. 1).

Beyersdorff et al. [13] were the first to describe a transrectal MRGB in a closed MR system by using anatomical T2-weighted MR images as part of a pre-biopsy planning. More recently, Hambrock et al. [15] and Franiel et al. [16] described transrectal MRGB in a closed MR system by using a multi-parametric MR pre-biopsy planning method. There are currently two commercially available devices for MRGB of the prostate. The devices have 5 degrees of freedom (rotation, forward and backward, change in height). Patients are placed in a prone or supine position on the MR table depending on the device. A fiducial (needle guider filled with gadolinium) is inserted rectally, after lubricating with lidocaine gel. A body phased-array coil is placed on the patient. Once the patient is correctly positioned and instructed, the CSR(s) determined during the diagnostic MR session need to be relocated. For this, these CSRs need to be translated to the T2-weighted MR images obtained during the biopsy session by using anatomical landmarks and a relative 3-dimensional position estimation [10]. To assess the most aggressive part of the CSR, a DWI sequence could be applied. Subsequently, the most restricted area could be targeted [19-21]. After relocation of the CSR the needle guider is adjusted toward the desired target area, a fast T2-weighted (for example, a true fast imaging with steady state precession sequence) verification image in at least two planes (preferably in the axial and the sagittal plane) is acquired to determine the needle guider's position relative to the targeted area. Both commercially available systems have software for correct alignment of needle guider and biopsy planning. When satisfied with its location a biopsy can be performed and a verification image with the needle left in situ can be obtained. An 18-gauge, fully automatic, MR-compatible, core needle, double-shot biopsy gun can be used for this purpose. Depending on how confident one is about the accuracy of the biopsy relative to the CSR, additional biopsies can be taken. A procedure time of $35 \mathrm{~min}$ is reachable, after a learning curve of probably $30-40$ cases.

Expected morbidity after MRGB are more or less comparable to random TRUSGB, which are hematospermia (for about 4 weeks after the procedure), hematuria (for about a week after the procedure), and anal bleeding (one or two days after the procedure). Sporadically sepsis can develop $(<1 \%)$. Even though it is conceivable that MRGB will have less complications because of the lower number of cores taken per patient, no studies report on the comparison of MRGB and TRUSGB complications.

\section{Discussion}

Detection rates for MRGB after at least one previous negative TRUS-guided biopsy session ranged between 38 and $59 \%[10,12-16]$. These rates are noticeably higher compared to repeat TRUS-guided biopsy, which has a detection rate of maximally $22 \%$ in the second session. Although TRUS-guided biopsy is more available worldwide compared to MRGB, the advantage of targeted biopsy over "blind" biopsy is clear. MRGB has the advantage of being a targeted procedure, directed by the preceding diagnostic MR session with promising localization performance. Also the excellent soft tissue contrast during the biopsy itself is a major advantage of this technique. The use of multi-parametric MR imaging in prostate cancer management could prevent patients from undergoing unnecessary biopsies and consequently avoid unnecessary treatment delay.

MR imaging findings have been used to direct biopsies under TRUS guidance with reasonable to good detection rates 25-55\%. Experimental fusion of MR and TRUS data [22], in which distances between corresponding data-points for each technique are rendered as small as possible by elastic registration, has been used to obtain more accurate MR-TRUS biopsy results.

A limitation of MRGB is that multi-parametric MR imaging for localization and the MR-guided biopsy need to be performed in two different sessions, as image postprocessing, reading and exact tumor localization demand time. Furthermore, antibiotics have to be administered before the biopsy session and pre-cautions regarding anticoagulation medication have to be taken. Another disadvantage is the movement of the prostate during the biopsy procedure and moving the patient in and out of the MR bore for device manipulation. The latter is time consuming and may increase the change of patient movement.

In conclusion, MRGB has a high prostate cancer detection rate in patients with previous negative TRUSguided biopsy sessions. For this reason MRGB and/or TRUS-MR fusion guided biopsy will probably become more and more available in daily practice.

Open Access. This article is distributed under the terms of the Creative Commons Attribution Noncommercial License which permits any noncommercial use, distribution, and reproduction in any medium, provided the original author(s) and source are credited.

\section{References}

1. Jemal A, Siegel R, Xu J, Ward E (2010) Cancer statistics, 2009. CA Cancer J Clin 60(5):277-300

2. Hricak H (2005) MR imaging and MR spectroscopic imaging in the pre-treatment evaluation of prostate cancer. Br J Radiol 78(2): S103-S111

3. Gilligan T, Kantoff PW (2002) Chemotherapy for prostate cancer. Urology 60:94-100

4. Thompson IM, Seay TM (1997) Will current clinical trials answer the most important questions about prostate adenocarcinoma? Oncology (Williston Park) 11:1109-1117

5. Thompson IM, Pauler DK, Goodman PJ, et al. (2004) Prevalence of prostate cancer among men with a prostate-specific antigen level $<$ or $=4.0 \mathrm{ng}$ per milliliter. N Engl J Med 350:2239-2246

6. Thompson IM, Ankerst DP, Chi C, et al. (2005) Operating characteristics of prostate-specific antigen in men with an initial PSA level of $3.0 \mathrm{ng} / \mathrm{ml}$ or lower. JAMA 294:66-70 
7. Gretzer MB, Partin AW (2003) PSA markers in prostate cancer detection. Urol Clin North Am 30:677-686

8. Canto EI, Singh H, Shariat SF, et al. (2004) Effects of systematic 12-core biopsy on the performance of percent free prostate specific antigen for prostate cancer detection. J Urol 172:900-904

9. Singh H, Canto EI, Shariat SF, et al. (2004) Improved detection of clinically significant, curable prostate cancer with systematic 12-core biopsy. J Urol 171:1089-1092

10. Hambrock T, Fütterer JJ Huisman HJ, et al. (2008) Thirty twochannel coil 3T magnetic resonance-guided biopsies of prostate tumor suspicious regions identified on multimodality 3T magnetic resonance imaging: technique and feasibility. Invest Radiol 43:686694

11. Hoeks CMA, Barentsz JO, Hambrock T, et al. (2011) Prostate cancer: multiparametric MR imaging for detection, localization, and staging. Radiology [Epub Ahead of Print].

12. Anastasiadis AG, Lichy MP, Nagele U, et al. (2006) MRI-guided biopsy of the prostate increases diagnostic performance in men with elevated or increasing PSA levels after previous negative TRUS biopsies. Eur Urol 50:738-748

13. Beyersdorff D, Winkel A, Hamm B, et al. (2005) MR imagingguided prostate biopsy with a closed MR unit at $1.5 \mathrm{~T}$ : initial results. Radiology 234:576-581

14. Engelhard K, Hollenbach HP, Kiefer B, et al. (2006) Prostate biopsy in the supine position in a standard 1.5-T scanner under real time MR-imaging control using a MR-compatible endorectal biopsy device. Eur Radiol 16:1237-1243

15. Hambrock T, Somford DM, Hoeks C, et al. (2010) Magnetic resonance imaging guided prostate biopsy in men with repeat negative biopsies and increased prostate specific antigen. J Urol 183:520-527
16. Franiel T, Stephan C, Erbersdobler A, Dietz E, et al. (2011) Areas suspicious for prostate cancer: MR-guided biopsy in patients with at least one transrectal US-guided biopsy with a negative finding - multiparametric MR imaging for detection and biopsy planning. Radiology 259:162-172

17. Hara R, Jo Y, Fujii T, et al. (2008) Optimal approach for prostate cancer detection as initial biopsy: prospective randomized study comparing transperineal versus transrectal systematic 12-core biopsy. Urology 71:191-195

18. Ingber MS, Ibrahim I, Turzewski C, Hollander JB, Diokno AC (2010) Does periprostatic block reduce pain during transrectal prostate biopsy? A randomized, placebo-controlled, double-blinded study. Int Urol Nephrol 42:23-27

19. Hambrock T, Somford DM, Huisman HJ, et al. (2011) Relationship between apparent diffusion coefficients at 3.0-T MR imaging and gleason grade in peripheral zone prostate cancer. Radiology 259:453-461

20. Mazaheri Y, Shukla-Dave A, Hricak H, et al. (2008) Prostate cancer: identification with combined diffusion-weighted MR imaging and 3D $1 \mathrm{H}$ MR spectroscopic imaging - correlation with pathologic findings. Radiology 246:480-488

21. de Souza NM, Riches SF, Vanas NJ, et al. (2008) Diffusionweighted magnetic resonance imaging: a potential non-invasive marker of tumour aggressiveness in localized prostate cancer. Clin Radiol 63:774-782

22. Singh AK, Kruecker J, Xu S, et al. (2008) Initial clinical experience with real-time transrectal ultrasonography-magnetic resonance imaging fusion-guided prostate biopsy. BJU Int 101:841-845 